\title{
Positron states in polypropylene and polystyrene at low temperature
}

\author{
N. Djourelov ${ }^{* 1,2}$, C. Dauwe ${ }^{1}$, C. A. Palacio ${ }^{1,3}$, N. Laforest ${ }^{1,4}$, and C. Bas ${ }^{4}$ \\ ${ }_{1}^{1}$ Department of Subatomic and Rad. Physics, Ghent University, Proeftuinstraat 86, 9000 Ghent, Belgium \\ 2 Institute of Nuclear Research and Nuclear Energy, 72 Tzarigradsko Shoosse, 1784 Sofia, Bulgaria \\ ${ }^{3}$ Institute of Physics, University of Antioquia, A. A. 1226, Medellin, Colombia \\ ${ }^{4}$ University of Savoie, Batiment IUT, 73376 Le Bourget-du-lac Cedex, France
}

Received 23 July 2006, accepted 27 March 2007

Published online 29 June 2007

PACS 61.41.+e, 71.60.+z, 78.70.Bj

The increase of the positronium (Ps) yield as a function of the positron $\left(\mathrm{e}^{+}\right)$source exposure time at $20 \mathrm{~K}$ was monitored in polypropylene and polystyrene by positron annihilation lifetime (PAL) and Doppler broadening (DB) spectroscopy. The contributions of the different $\mathrm{e}^{+}$and Ps states as extracted from the PAL analysis were used to reproduce the behaviour of the sharpness parameter $(S)$ as a function of the wing parameter $(W)$ of the annihilation line as measured by DB spectroscopy. We find a remarkable nonlinearity in the $S$ - $W$ plots, which seems to be related to the existence of a third component $\left(\mathrm{C}_{3}\right)$ in a fourcomponent exponential analysis. We discuss the origin of $\mathrm{C}_{3}$ in the frame of a "blob" analysis of the PAL spectra. It is shown that the simulated $S$ - $W$ dependence can satisfactorily fit the observed non-linearity only on the assumption that $\mathrm{C}_{3}$ characterizes a $\mathrm{e}^{+}$-molecule complex.

(C) 2007 WILEY-VCH Verlag GmbH \& Co. KGaA, Weinheim

1 Introduction It is widely reported that positron annihilation lifetime (PAL) spectra of various polymers can be fitted with three exponential components. The obtained intensities ratio of orthoPositronium (oPs) to para-Positronium (pPs) usually is unacceptably lower than the statistical prediction of 3. Better fit gives a four exponential analysis. While there is an agreement that the longest-lived component comes from the pick-off annihilation of oPs confined in free-volume holes (FVH) in the amorphous regions of a polymer, the origin of the shorter long-lived component $\left(\mathrm{C}_{3}\right)$ is still a subject of discussions [1]. The annihilation line is Doppler broadened due to the momentum of the annihilating pair along the direction of the detection by a HPGe-detector (DB technique). The extent of the broadening is usually evaluated by the peak shape $(S)$ and wing $(W)$ parameters. Despite of their integral character, a simultaneous consideration of the changes in $S$ and $W$ and the PAL components characteristics could supply valuable information about the $\mathrm{C}_{3}$ origin.

2 Experimental Additive-free isotactic polypropylene (PP) with $66 \%$ crystallinity and amorphous atactic polystyrene (PS) were studied. As a positron source we used $1.5 \mathrm{MBq}{ }^{22} \mathrm{NaCl}$ packed in $7-\mu \mathrm{m}-$ thick Kapton. The source-sample sandwich mounted on the cold finger of a cryostat was cooled in $\sim 1 \mathrm{~h}$ down to $20 \mathrm{~K}$. The PAL spectrometer showed resolution of $230 \mathrm{ps}$ full width at half maximum (FWHM). For the DB measurements, a HPGe detector with energy resolution of $\mathrm{G}_{1 / 2}=1.06 \mathrm{keV}$ (FWHM of the 514-keV line of ${ }^{85} \mathrm{Sr}$ ) was used. The PAL and DB spectra were recorded at every $2 \mathrm{~h}$ with statistics respectively of $\sim 9 \times 10^{5}$ and $8.5 \times 10^{6}$ (in the peak). The spectra for PS were summed in groups of four. The $511-\mathrm{keV}$ photo peak was characterized by the $S$ parameter defined as the sum of the counts in the central

\footnotetext{
" Corresponding author: e-mail: nikdjour@inrne.bas.bg, Phone: +359 2714 308, Fax: +359 29744978
} 
region $(|\Delta E|<0.7 \mathrm{keV})$ of the peak relative to the total peak counts $\left(N_{\text {tot }}\right)$ and the $W$ parameter defined as the ratio of the sum of the counts in the photo peak wings $(2.5<|\Delta E|<7 \mathrm{keV})$ to $N_{\text {tot }}$.

3 Results and discussion The general explanation for the $S$ parameter composed of a free $\mathrm{e}^{+}\left(S_{+}\right)$ and one or more trapped Ps states, which annihilate by self annihilation $\left(S_{\mathrm{sa}}\right)$ or pick-off annihilation $\left(S_{\mathrm{po}}\right)$, is given by

$$
S=(1 / 4) \sum I_{\mathrm{Ps}}^{i}\left[\beta^{i} S_{\mathrm{sa}}^{i}+\left(1-\beta^{i}\right) S_{\mathrm{po}}^{i}\right]+\left(1-\sum I_{\mathrm{Ps}}^{i}\right) S_{+}+(3 / 4) \sum I_{\mathrm{Ps}}^{i} S_{\mathrm{po}}^{i},
$$

where $I_{P s}^{i}$ is the yield of the $i$-th Ps with pPs self annihilation branching ratio of $\beta^{i}$.

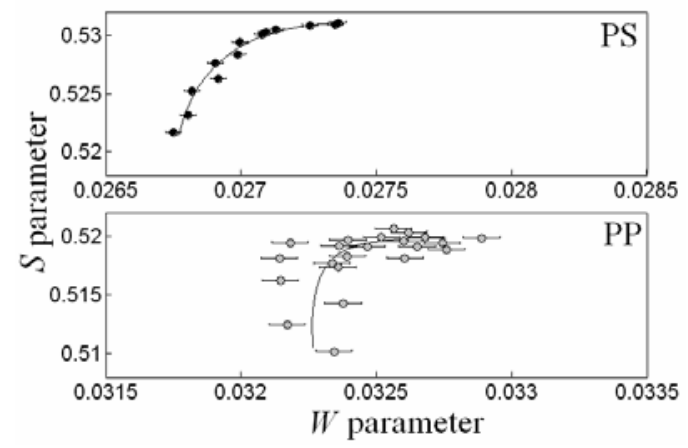

Fig. $1 S$ as a function of $W$ for PS and PP. The lines represent a simulation based on the PAL analysis.

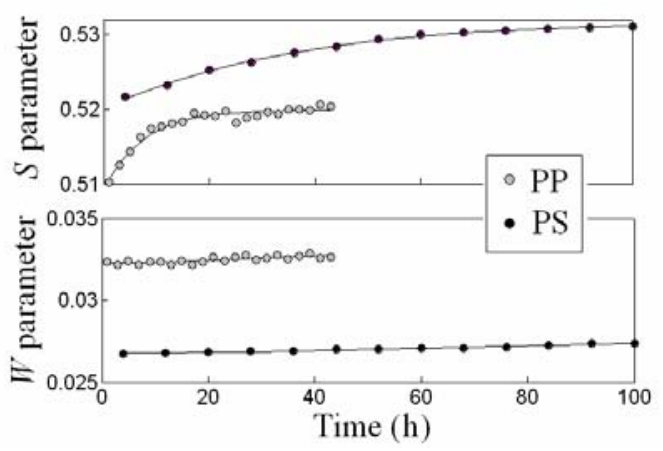

Fig. $2 S$ and $W$ as a function of the source exposure time, $t_{\mathrm{m}}$, for PS and PP.

By ignoring the time dependence of the fast intrablob processes the differential equations which describes the kinetics of the $\mathrm{e}^{+}$states can be easily solved analytically. Then in the case of multiple types of $\mathrm{FVH}$, the oPs intensities are

$$
I_{\mathrm{oPs}}^{i}=(3 / 4)\left\{k_{\mathrm{bl}} /\left(\lambda_{+}-\lambda_{\mathrm{po}}^{i}+k_{\mathrm{bl}}\right)\right\}\left[v_{\mathrm{Ps}}^{i} /\left(\lambda_{+}-\lambda_{\mathrm{po}}^{i}+\sum v_{\mathrm{Ps}}^{i}\right)\right],
$$

where $v_{\mathrm{Ps}}^{i}$ are the corresponding Ps trapping rates, $\lambda_{+}$is the free $\mathrm{e}^{+}$annihilation rate, and $k_{\mathrm{bl}}$ is the formation rate of nonlocalized Ps (qPs) from $\mathrm{e}^{+}$and intrablob $\mathrm{e}^{-}$[2]. It has been proven that there is no direct relation between the Ps yield and FVH concentration in polymers [3], i.e. we can consider that the case $v_{\mathrm{Ps}}^{i} » \lambda_{+}, k_{\mathrm{b} 1} » \lambda_{\mathrm{po}}^{i}$ described in [2] is also valid for our samples. Hence, Eq. (2) can be written as

$$
I_{\mathrm{Ps}}^{i}=I_{\mathrm{Ps}} v_{\mathrm{Ps}}^{i} / \sum v_{\mathrm{Ps}}^{i} .
$$

This formula expresses the state branching of qPs accepted in Ref. [2] as the precursor of Ps and is still valid if some of the traps are not FVH but molecule centers. Actually, the formula remains valid if the finite recombination time of the blob is taken into account, as well. From Eqs. (1) and (3) follows that $S$ should be a linear function of $I_{\mathrm{Ps}}$. Analogously for $W$, and, thus, the $S-W$ plot should be a straight line. The advantage of the $S-W$ presentation is that the uncertainty in determination of $I_{\mathrm{Ps}}$ is avoided. Obviously, the experimental $S$ - $W$ plots for PP and PS (Fig. 1) are far from any linearity. Consequently, an additional annihilation channel exists and it has to be considered as another $\mathrm{e}^{+}$state, trapped $\mathrm{e}^{+}$or $\mathrm{e}^{+}-$ molecule complex $\left(\mathrm{Me}^{+}\right)$.

Recently, Dauwe et al. have shown that the spatial and time dependences of the blob can be properly coded to compute the populations of the $\mathrm{e}^{+}$and Ps states [4]. By averaging the blob spatial distribution the recombination of the blob electrons can be described by $\rho_{-}(t)=\rho_{-}(0) \exp \left(-t / \tau_{\mathrm{bl}}\right)$, where $\tau_{\mathrm{bl}}$ is the blob recombination lifetime and $\rho_{-}(0)$ is the number of excess electrons in the blob induced by one $\mathrm{e}^{+}$. The normalized background-free PAL spectrum can be presented as $Y(t)=R(t) \otimes \sum \lambda_{i} \rho_{i}(t)$, where $\rho_{i}$ are the 
populations of the $\mathrm{e}^{+}$and Ps states, $R(t)$ is the PAL resolution function and $i$ runs the indexes for free $\mathrm{e}^{+}$, pPs, oPs, $\mathrm{Me}^{+}$(index ' $c$ '). The kinetics of the populations is described by the following set of differential equations

$$
\begin{array}{ll}
d \rho_{\mathrm{pPs}}(t) / d t=(1 / 4) k_{\mathrm{Ps}} \rho_{-}(t) \rho_{+}(t)-\lambda_{\mathrm{pPs}} \rho_{\mathrm{pPs}}(t) & \rho_{\mathrm{pPs}}(0)=0 \\
d \rho_{\mathrm{oPs}}(t) / d t=(3 / 4) k_{\mathrm{Ps}} \rho_{-}(t) \rho_{+}(t)-\lambda_{\mathrm{po}} \rho_{\mathrm{oPs}}(t), \text { with initial populations } & \rho_{\mathrm{oPs}}(0)=0, \\
d \rho_{\mathrm{c}}(t) / d t=v_{\mathrm{c}} \rho_{+}(t)-\lambda_{\mathrm{c}} \rho_{\mathrm{c}}(t) & \rho_{\mathrm{c}}(0)=0 \\
d \rho_{+}(t) / d t=-\left[\lambda_{+}+v_{\mathrm{c}}+k_{\mathrm{Ps}} \rho_{-}(t)\right] \rho_{+}(t) & \rho_{+}(0)=1
\end{array}
$$

where $k_{\mathrm{Ps}}$ is the Ps formation rate. Initially we have coded a system of the kinetic equations which describes a two-step Ps formation process, i.e. formation of qPs followed by trapping. The results of the analysis of various spectra have shown that the condition $v_{\mathrm{Ps}}^{i} » \lambda_{+}, k_{\mathrm{bl}} » \lambda_{\mathrm{po}}^{i}$ is fulfilled resulting also in computed $I_{\mathrm{qPs}}<1 \%$. Therefore, by the first equation of Eqs. (4) we have described one step Ps formation process. By applying a standard fitting procedure with incorporated numerical integration we fitted Eqs. (4) to the measured PAL spectra for the set of parameters $\left[\tau_{\mathrm{bl}}, v_{\mathrm{c}}, \zeta_{\mathrm{Ps}}, \lambda_{+}, \lambda_{\mathrm{c}}, \lambda_{\mathrm{po}}\right]\left(\zeta_{\mathrm{Ps}}\right.$ notes $\left.\rho_{-}(0) k_{\mathrm{Ps}}\right)$ with fixed $\lambda_{\mathrm{pPs}}=1 / 0.125 \mathrm{~ns}^{-1}$ plus the parameters for $R(t)$. The best fit gave $\left[\lambda_{+}, \lambda_{\mathrm{c}}, \lambda_{\mathrm{po}}\right]=[1 / 0.374,1 / 0.579$, $1 / 1.690]$ in $\mathrm{ns}^{-1}$ for PS and [1/0.369, 1/0.702, 1/1.600] for PP.

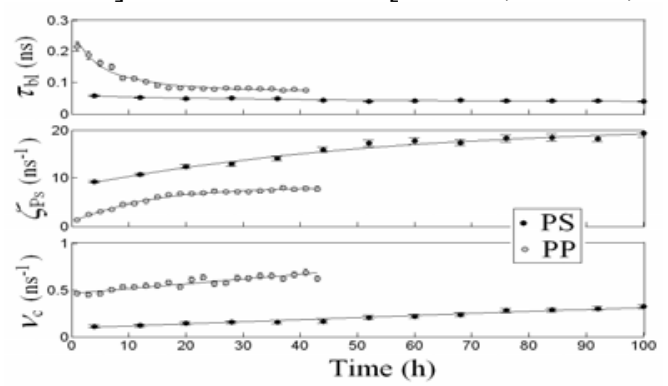

Fig. 3 Blob results for $\mathrm{e}^{+}$trapping rate, $v_{\mathrm{c}}$, Ps formation rate, $\zeta_{\mathrm{Ps}}$, and the blob recombination lifetime, $\tau_{\mathrm{bl}}$, as a function of the source exposure time, $t_{\mathrm{m}}$.

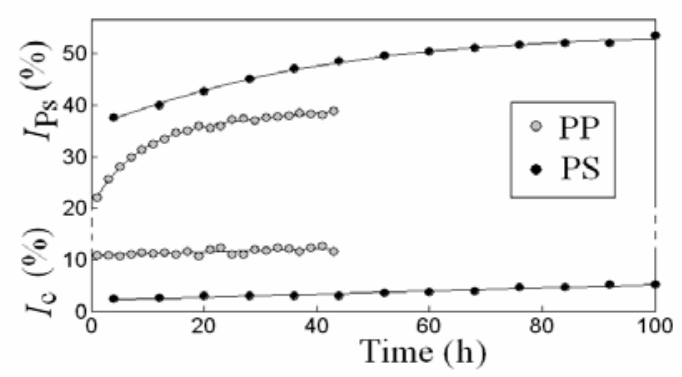

Fig. 4 Intensities $I_{\mathrm{c}}$ and $I_{\mathrm{Ps}}$, calculated from the blob analysis of the PAL spectra, as a function of the source exposure time, $t_{\mathrm{m}}$.

The best fit parameters, $\tau_{\mathrm{bl}}, v_{\mathrm{c}}$, and $\zeta_{\mathrm{Ps}}$, are presented in Fig. 3 . We see an increase of $\zeta_{\mathrm{Ps}}$ with $t_{\mathrm{m}}$. The enhanced Ps formation at low temperatures in polymers is due to accumulation of shallowly trapped electrons $\left(\mathrm{e}_{\mathrm{sh}}^{-}\right)$which concentration as a function of $t_{\mathrm{m}}$ can be expressed as [5]

$$
\rho_{\mathrm{sh}}\left(t_{\mathrm{m}}\right)=\rho_{\mathrm{sh}}^{0}\left[1-\exp \left(-t_{\mathrm{m}} / \tau_{\mathrm{d}}\right)\right]
$$

where $\rho_{\mathrm{sh}}^{0}=J \omega_{\mathrm{d}}^{-1} \exp \left(E / k_{\mathrm{B}}\right), J$ is the $\mathrm{e}_{\mathrm{sh}}^{-}$concentration induced by $\mathrm{e}^{+}$in unit time, $\tau_{\mathrm{d}}=\omega_{\mathrm{d}}^{-1} \exp \left(E / k_{\mathrm{b}} T\right)$ is the characteristic time, $\omega_{\mathrm{d}}$ is the typical oscillation frequency, $E$ is the activation energy of detrapping, and $k_{\mathrm{B}}$ is the Boltzmann's constant. If we assume that Ps can be formed at any $\mathrm{e}^{+}$age then in Eqs. (4) the term $k_{\mathrm{Ps}} \rho_{-}(t)$ has to be replaced by $k_{\mathrm{Ps}} \rho_{-}(t)+k_{\mathrm{Ps}}^{\mathrm{sh}} \rho_{\mathrm{sh}}$, where $k_{\mathrm{Ps}}^{\mathrm{sh}}$ is the reaction constant of $\mathrm{e}^{+}$and $\mathrm{e}_{\mathrm{sh}}^{-}$to form Ps. The last assumption is based on the age-momentum correlation (AMOC) experiments which indicate young age broadening due to delayed Ps formation [6]. However, the fitting results (not presented), based on this assumption, did not lead to any acceptable behaviour of the fitting parameters as a function of $t_{\mathrm{m}}$. Dauwe et al. have shown that the delayed Ps formation observed in AMOC experiments can excellently be explained by a finite blob recombination time [4]. Thus, we have accepted that the Ps formation occurs only during the blob existence, what formally means to replace the term $k_{\mathrm{Ps}} \rho_{-}(t)$ in Eqs. (4) by $k_{\mathrm{Ps}} \rho_{-}(t)+k_{\mathrm{Ps}}^{\mathrm{sh}} \rho_{\mathrm{sh}} \exp \left(-t / \tau_{\mathrm{bl}}\right)$. From Eq. (5) follows that $\zeta_{\mathrm{Ps}}\left(t_{\mathrm{m}}\right)=\zeta_{\mathrm{Ps}}^{\mathrm{bl}}+\zeta_{\mathrm{Ps}}^{\mathrm{sh}}\left[1-\exp \left(-t_{\mathrm{m}} / \tau_{\mathrm{d}}\right)\right]$, where $\zeta_{\mathrm{Ps}}^{\mathrm{sh}}=\rho_{\mathrm{sh}}^{0} k_{\mathrm{Ps}}$, and $\zeta_{\mathrm{Ps}}^{\mathrm{bl}}, \zeta_{\mathrm{Ps}}^{\mathrm{sh}}$, and $\tau_{\mathrm{d}}$ are found being $0.9 \mathrm{~ns}^{-1}, 7.2 \mathrm{~ns}^{-1}, 14 \mathrm{~h}$ for PP and $8.1 \mathrm{~ns}^{-1}, 13$ $\mathrm{ns}^{-1}, 55 \mathrm{~h}$ for PS. The lower $\zeta_{\mathrm{Ps}}^{\mathrm{bl}}$ and $\zeta_{\mathrm{Ps}}^{\mathrm{sh}}$ values for PP are probably due to the fact that PP is semicrystalline while PS is amorphous. Another result seen in Fig. 3 is that $v_{\mathrm{c}}$ slightly increases linearly with $t_{\mathrm{m}}$ 
with slope of $0.0022 \mathrm{~h}^{-1}$ and $0.0051 \mathrm{~h}^{-1}$ for PS and PP. This means that the number of the trapping centres slightly increases with $t_{\mathrm{m}}$. The radicals created by the $\mathrm{e}^{+}$irradiation may have negative or positive electron affinity. The former may directly attach $\mathrm{e}^{+}$to form $\mathrm{Me}^{+}$while the last ones first may react with $\mathrm{e}^{-}$to form an anion radical and then to trap $\mathrm{e}^{+}$. The same additives-free PP contains detectably high, by means of coincidence DB technique, initial concentration of carbonyl groups [7]. The oxygen in such groups is partly negatively charged and is an excellent candidate to trap $\mathrm{e}^{+}$. It seems that such $\mathrm{e}^{+}$trapping centres exist a priori to the $\mathrm{e}^{+}$irradiation in PP but not in PS. The change in $v_{\mathrm{c}}$ with $t_{\mathrm{m}}$ is in agreement that in a PP oxidation, stimulated by the $\mathrm{e}^{+}$irradiation, is weak but possible at low temperature [8]. Both time constant $\tau_{\mathrm{d}}$ and slope of the change in $v_{\mathrm{c}}\left(t_{\mathrm{m}}\right)$ indicate that $\mathrm{e}^{+}$irradiation results in faster changes in PP than in PS. This agrees with the data on radiation stability of polymers that generally PS is more stable than PP. On the assumption that the additional state is a $\mathrm{Me}^{+}$state, Eq. (1) takes the form:

$$
\left.S\left(t_{\mathrm{m}}\right)=\left(I_{P_{s}}\left(t_{\mathrm{m}}\right) / 4\right) \mid \beta S_{\mathrm{sa}}+(1-\beta) S_{\mathrm{po}}\right\rfloor+\left(1-I_{\mathrm{c}}-I_{P_{s}}\left(t_{\mathrm{m}}\right)\right) S_{+}+I_{\mathrm{c}}\left(t_{\mathrm{m}}\right) S_{\mathrm{c}}+(3 / 4) I_{P s}\left(t_{\mathrm{m}}\right) S_{\mathrm{po}},
$$

where $\beta=\eta \lambda_{\mathrm{pPs}}^{0} /\left(\eta \lambda_{\mathrm{pPs}}^{0}+\lambda_{\mathrm{po}}\right), \eta=0.7$ is the Ps contact density, and $\lambda_{\mathrm{pPs}}^{0}=1 / 0.125 \mathrm{~ns}^{-1}$ is the pPs self annihilation rate, and $I_{\mathrm{c}}$ and $I_{\mathrm{Ps}}$, shown in Fig. 4 , are computed from the blob results by a posteriori integration of the corresponding contributions [4]. There is a similar equation (6') for $W$. The narrow Gaussian in the decomposition of the annihilation pair momentum is due to self annihilation of pPs confined in FVH. We computed $S_{\mathrm{sa}}=0.764 ; 0.761$ and $W_{\mathrm{sa}}=0.000070 ; 0.000057$ for PP and PS as the parameters of the corresponding Gaussian with FWHM $=\left[\left(\theta_{1 / 2}\right)^{2}+\left(\mathrm{G}_{1 / 2}\right)^{2}\right]^{0.5}$ by applying the well-known Tao-Eldrup formula for calculation of $R$ from $\lambda_{\mathrm{po}}$ and then $\theta_{1 / 2}$ from $R$. So, we fitted Eqs. (6) and (6') to experimental $S\left(t_{\mathrm{m}}\right)$ and $W\left(t_{\mathrm{m}}\right)$. The best fits are shown as lines in Figs. 1 and 2 for the following sets of best fit parameters $S_{+}=0.504 ; 0.496, S_{\mathrm{c}}=0.44 ; 0.46, S_{\mathrm{po}}=0.509 ; 0.507$ and $W_{+}=0.0272 ; 0.0266, W_{\mathrm{c}}=0.076 ; 0.054$, $W_{\mathrm{po}}=0.034 ; 0.033$ for PP and PS, correspondingly. For both PP and PS the $S_{\mathrm{c}}$ shows low value and $W_{\mathrm{c}}$ high value which is a clear indication that from the $\mathrm{Me}^{+}$state $\mathrm{e}^{+}$annihilates with greater probability with carbon and/or oxygen core $\mathrm{e}^{-}$than the free $\mathrm{e}^{+}$. Obviously, the change in $S\left(t_{\mathrm{m}}\right)$ is dominated by $I_{\mathrm{Ps}}$ while this in $W\left(t_{\mathrm{m}}\right)$ is dominated by $I_{\mathrm{c}}$.

4 Conclusions We have based our considerations on a set of differential equations which describe the kinetics of $\mathrm{e}^{+}$and Ps states. The observed non-linearity in the $S-W$ plots suggests that the additional state has to be due to annihilation of $\mathrm{Me}^{+}$complex. The contributions of the different $\mathrm{e}^{+}$and Ps states as extracted from the PAL blob analysis have been used to reproduce the behaviour of the experimental $S$ and $W$ as a function of the source exposure time. The best fit parameters for $S_{\mathrm{c}}$ and $W_{\mathrm{c}}$ of $\mathrm{Me}^{+}$showed high momentum of the corresponding annihilation pair. The last confirms that $\mathrm{Me}^{+}$state is distinguishable from the free $\mathrm{e}^{+}$state. The $\mathrm{Me}^{+}$state seems to be associated to $\mathrm{e}^{+}$trapping by oxygen containing groups and/or free radicals.

Acknowledgements This research was supported by the IAP program P5/01 of the Belgian Science Policy Office. (BELSPO). The first author (N.D.) is grateful for the research fellowship awarded by BELSPO(FEDRA).

\section{References}

[1] G. Dlubek and S. Eichler, phys. stat. sol. (a) 168, 333 (1998).

[2] V. Shantarovich, T. Suzuki, C. He, V. Davankov, A. Pastukhov, K. Kondo, Y. Ito, and M. Tsurupa, Radiat. Phys. Chem. 68, 639 (2003).

[3] F. H. J. Maurer and M. Schmidt, Radiat. Phys. Chem. 58, 509 (2000).

[4] C. Dauwe, B. Van Waeyenberge, and N. Balcaen, Phys. Rev. B 68, 132202 (2003).

[5] C. He, T. Suzuki, V. P. Shantarovich, L. Ma, M. Matsuo, K. Kondo, and Y. Ito, Phys. Lett. A 313, 223 (2003).

[6] N. Suzuki, T. Hirade, F. Saito, and T. Hyodo, Radiat. Phys. Chem. 68, 647 (2003).

[7] N. Djourelov, C. He, T. Suzuki, V. Shantarovich, Y. Ito, K. Kondo, and Y. Ito, Radiat. Phys. Chem. 68, 689 (2003).

[8] N. Djourelov, T. Suzuki, R. S.Yu, V. Shantarovich, and K. Kondo, Chem. Phys. 302, 179 (2004). 